\title{
Labyrinthine and Cortical Concussion: Treatment with Vestibular and Cognitive Rehabilitation
}

\author{
Gans RE and Darren Kurtzer* \\ The American Institute of Balance, Largo, Florida
}

Submission: March 02, 2016; Published: March 09, 2017

*Corresponding author: Darren Kurtzer, The American Institute of Balance, Largo, Florida, USA, Tel: 727-398-5728; Email: dkurtzer@dizzy.com

Abstract

The gelatinous brain freely floating in the skull can have multiple impact points during head trauma. On the same side of the impact is the coup injury to the brain; on the opposite side of the impact is the contra-coup injury; and the vectors of force that travel through the entire brain can also cause tearing or shearing of neurons, leading to injury. The American Institute of Balance - Vestibular Cognitive Integration (AIB-VCI) recognizes research showing that creating too much cognitive demand, including during therapy sessions, can have devastating and long-lasting effects on the cognitive abilities of the patient, suggesting that adequate rest periods can be of utmost importance in terms of an efficacious rehabilitation strategy [1]. Thus, being able to independently adjust for cognitive and vestibular activities leads to a safe and effective means of cognitive and vestibular rehabilitation post-concussion.

Abbreviations: AIB-VCI: American Institute of Balance - Vestibular Cognitive Integration; TBI: Traumatic Brain Injury; RTA: Return-ToActivity; BVMT: Brief Visuospatial Memory Task; HVLT: Hopkin’s Verbal Learning Task; SDMT: Symbol Digit Modalities Test

\section{Introduction}

There has been an increase in the rate of traumatic brain injury (TBI) occurrence from 2001 through present of approximately 824 patients per 100,000 (CDC, 2016). Concussions were thought to only involve a specific set of symptoms; namely, unconsciousness, memory impairment, or confusion [2]. As we are now aware, concussions can be much more diverse than the aforementioned symptoms suggest. The gelatinous brain freely floating in the skull can have multiple impact points during head trauma. On the same side of the impact is the coup injury to the brain; on the opposite side of the impact is the contra-coup injury; and the vectors of force that travel through the entire brain can also cause tearing or shearing of neurons, leading to injury. The gelatinous nature of the brain makes it particularly susceptible to these shearing and tearing forces [3].

This kind of damage can impair the metabolic functions of neurons causing them to struggle to release, reabsorb, or utilize neurotransmitters at the same rate they did prior to being damaged. This leads to metabolic strain of the impaired neurons and allows for an increased amount of byproducts to build up inside of the nerve cell. These byproducts are toxic to cells at high levels. For example, impaired calcium metabolism can lead to cellular death post-concussion [4]. When a nerve cell dies, the linking cells mustcompensate for the lost neuronal activity. This can result in added demand and cause the cycle of byproduct build-up to occur in the once healthy nerve cells, leading to cell death in the extreme case of overuse.

Since no surgical intervention, or pharmacological management can heal nerve cells, low-level cognitive activity, or cognitive rest, is one of the best recommendations to ensure proper nerve cell recovery [5]. Due to the idiopathic nature of concussions, individual and graduated return-to-activity (RTA) management that incorporates initial cognitive rest may prove to be the most beneficial [6]. As recent research has shown that a human nerve cell can die within 6-hours post-concussive force [7], there should be a minimum of 6 hours of cognitive rest post impact to ensure the best possible chance of healing. If nerve cells are not given adequate rest, then proper healing may not occur, and the added cognition of daily tasks, or therapeutic intervention, may further impair nerve function. One hour of intense cognition daily has been shown to be a safe strategy to reduce the likelihood of placing too much demand on nerve cells and causing further impairment [8].

The goal of this protocol is to incorporate clinicians and patients in an integrated approach to achieve successful vestibular and concussion management. The protocol utilizes psychometrically analyzed cognitive assessment and management tools and integrates them with well-established vestibular rehabilitation protocols developed at The American 
Institute of Balance. This allows for longitudinal assessment as well as an objective RTA decision at the program's conclusion. We present the following patient as an example of the usefulness of the protocol at rehabilitating both cortical and labyrinthine aspects of concussion.

\section{History}

A 46-year-old female was seen at our facility for concussion management. Shereports sustaining a head injury during a motor vehicle accident 3 weeks ago. The impact to the head occurred on the left frontal area. The patient noticed immediate vertigo post-impact, lasting for approximately 24-hours. Currently, she remarks of blurred vision with active head rotation as well as difficulty ambulating on dynamic surface. She reports brief vertigo episodes when laying down at night. The patient also notices short-term memory impairment, especially for numbers. She gives the example of having problems remembering phone numbers at work, which used to be a regular task pre-impact. She states that paying attention to people speaking, or written instructions on the computer screen for more than a few minutes is now an issue for her. When the patient attends her regular exercise class, she finds the experience overwhelming specifically when her heart rate begins to increase. Of note, CT and MRI studies performed post-impact were negative for significant findings.

\section{Vestibular Assessment}

Hallpike and positional testing: positive for bilateral posterior canal benign paroxysmal positional vertigo (PC-BPPV), subjectively worse on the left side
a) Gaze testing: No nystagmus
b) Rotary Chair testing: Asymmetric gain to step velocity leftward

c) Head Thrust: corrective saccades with leftward head thrusts

d) Optokinetic studies: Symmetric gain bidirectionally

e) Gans SOP testing: fall on condition 6, right turn on condition 7

\section{Cognitive Assessment}

Woodcock-Johnson Tests of Cognitive Abilities showing deficits:
a) Block rotation
b) Retrieval fluency
c) Numbers reversed
d) Verbal attention

AIB - Vestibular-Cognitive Integration Assessment and Management Tools
a) Brief Visuospatial Memory Task (BVMT)
b) Hopkin's Verbal Learning Task (HVLT)
c) NAB Digits Forward/Backward
d) Stroop Test
e) Symbol Digit Modalities Test (SDMT)

\section{Management Strategy}

I. Canalith repositioning: left PC first, then right PC

II. VRT for uncompensated left vestibulopathy using hybrid techniques for: substitution, gaze stabilization, adaptation, and habituation protocols focusing on $0.5-1 \mathrm{~Hz}$ head movements

III. Cognitive management strategies:

a) Block rotation

b) Retrieval fluency

c) Numbers reversed

d) Verbal attention

A. Patient should reduce cognitive load to 1 hour of cognition daily

B. Vitamin C at $1-2,000 \mathrm{mg}$ daily in AM or after physical activity to reduce cortisol

C. Vitamin E at up to 1,000 mg daily as neuro and vascular protectant

D. Omega 3-6-9 supplementation (either increasing 3 with supplementation or decreasing 6 in diet) for neuro and vascular protectant

\section{Management Protocol (Figure 1)}

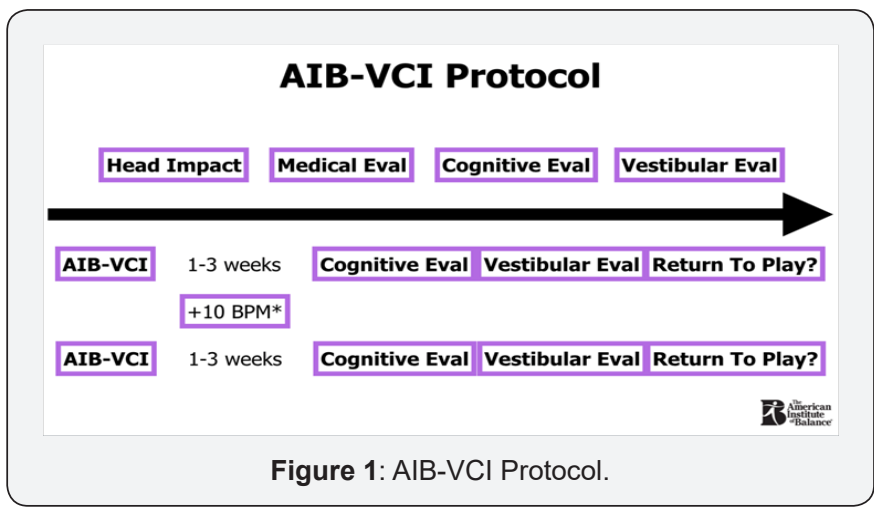

I. Our protocol consists of a combination of vestibular rehabilitation exercises and cognitive training exercises. The former consists of adaptation, habituation, gaze stabilization, and substitution based on the patient's symptoms and test findings; the latter consists of training material that relates to the cognitive tests that show deficit. Due to the complaint 
of exercising provoking symptoms, heart rate (HR) will be incorporated in the rehabilitation process (Figure 2).

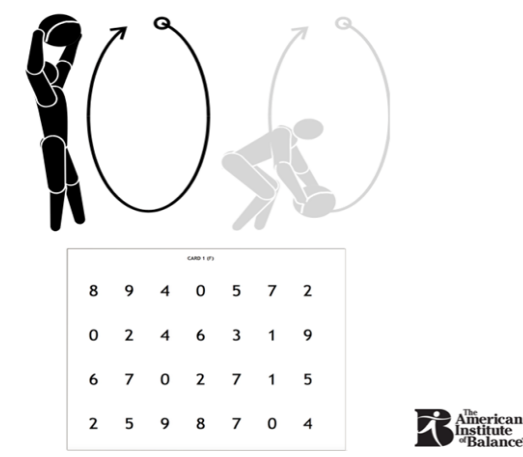

Figure 2: Heart rate vs Exercises.

II. One of the selected exercises for the patient based on her vestibular complaints of oscillopsia with active head rotation as well as her cognitive complaints of digit memory issues. During this exercise, the patient would make a circle pattern of movement with her body and head while focusing on a ball with a cue card attached, or with the cue card alone, in addition to performing a forward/backward digit memory task.

\section{Dynamic Functional Reach}

Begin with both feet flat on the ground at shoulder width

The therapist will move the iPad or cards around the patient such that they must bend/stretch/reach for the correct

answer without moving their feet from

the starting position (this example uses Spatial Awareness Test protocols)

As the patient progresses, they may stand on a dynamic surface or begin in a single leg stance

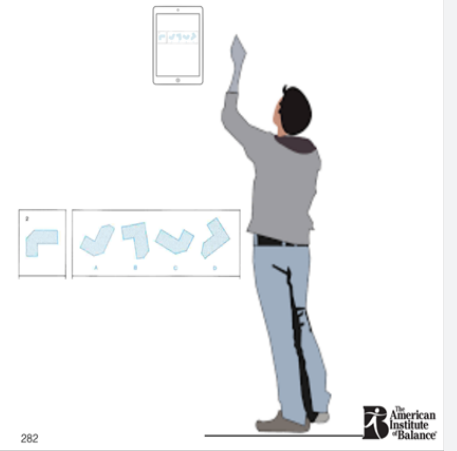

Figure 3: Dynamic Functional Reach.

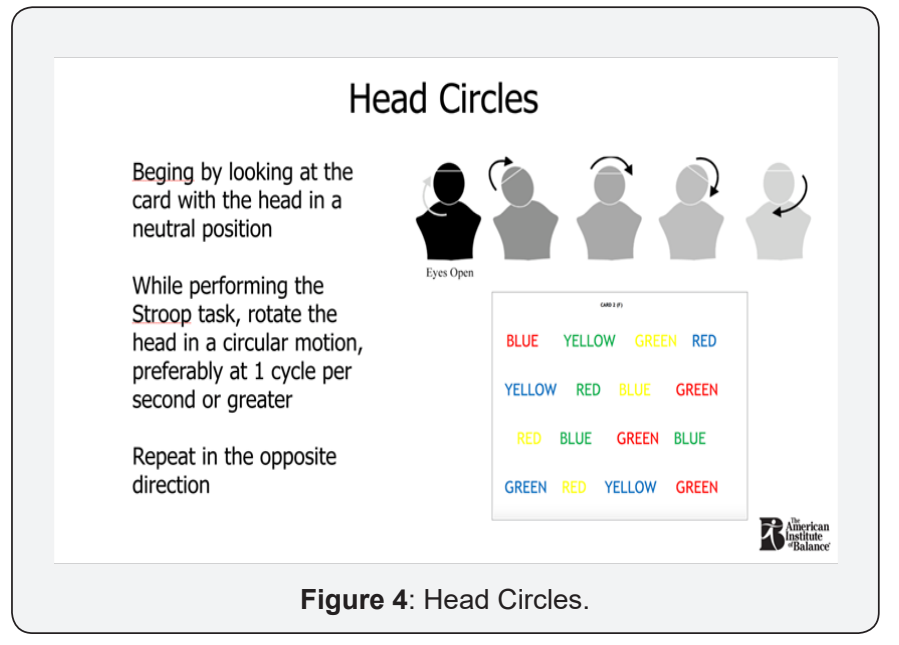

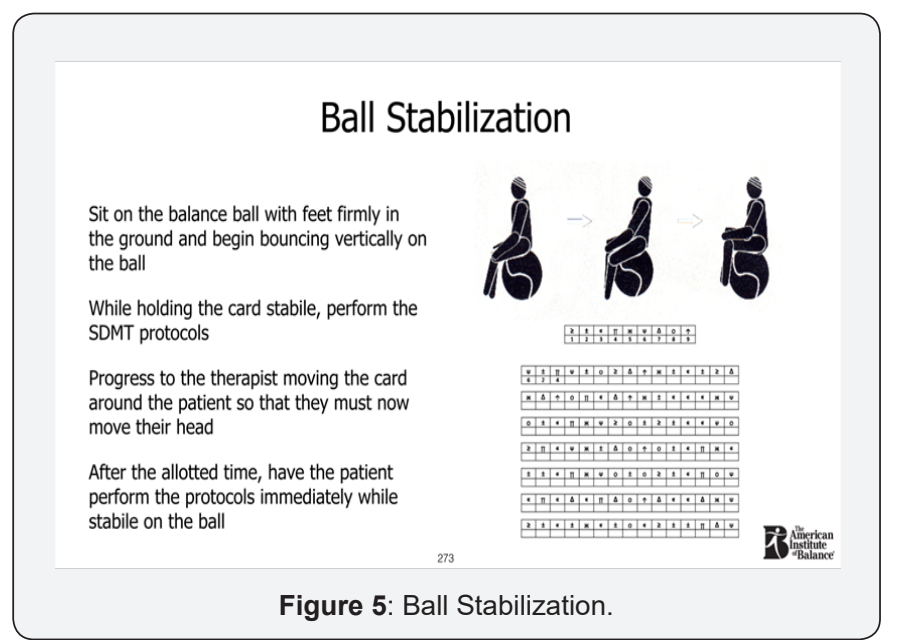

Following are more examples of AIB-VCI exercises used with the patient (Figures 3-5). All of the exercises were completed 3-5 days per week for one hour per session. Of the 3-5 days per week sessions, 2 of them were done with elevated heart rate. Our protocol, adapted from the Buffalo Concussion Treadmill test $[9,10]$, required the patient to first elevate her heart rate to her symptom-limited maximum (88 BPM in this case). Then, $80 \%$ of this value was used as a starting point, gradually progressing to her target of 100 BPM. During the heart rate training sessions, the patient completed the AIB-VCI exercises as described previously after elevating her heart rate to $80 \%$ of the symptomlimited maximum. Every 3 weeks, the heart rate was increased by $10 \mathrm{BPM}$ if her vestibular and cognitive scores were improving, as measured by the 3-week re-assessment period.
A. First Follow-up
a) Vestibular assessment
i. Hallpike and positional testing: negative for BPPV bilaterally
ii. Gaze testing: No nystagmus
iii. Rotary Chair testing: Symmetric gain to step velocity bidirectionally

iv. Head Thrust: corrective saccades with leftward head thrusts

v. Optokinetic studies: Symmetric gain bidirectionally

vi. Gans SOP testing: fall on condition 6

b) Cognitive assessment

I. AIB - Vestibular-Cognitive Integration Assessment and Management Tools

i. Brief Visuospatial Memory Task (BVMT) - Improvement

ii. Hopkin's Verbal Learning Task (HVLT) - Decline

iii. NAB Digits Forward/Backward - Improvement

iv. Stroop Test - Improvement 
v. Symbol Digit Modalities Test (SDMT) - Decline

\section{Management Strategy}

a. VRT for uncompensated left vestibulopathy

using hybrid techniques for: substitution, gaze stabilization, adaptation, and habituation protocols focusing on $1 \mathrm{~Hz}-1.5 \mathrm{~Hz}$ head movements

b. Cognitive management strategies:

i. Since SDMT and HVLT declined, we need to either reduce the time spent on the cognitive exercises or reduce the cognitive load

ii. This was done by reducing the difficulty of the cognitive tasks as well as the paired vestibular tasks

iii. Heart rate will be increased by 10 BPM for all exercises except those using adaptations from the SDMT and HVLT (Figure 6)

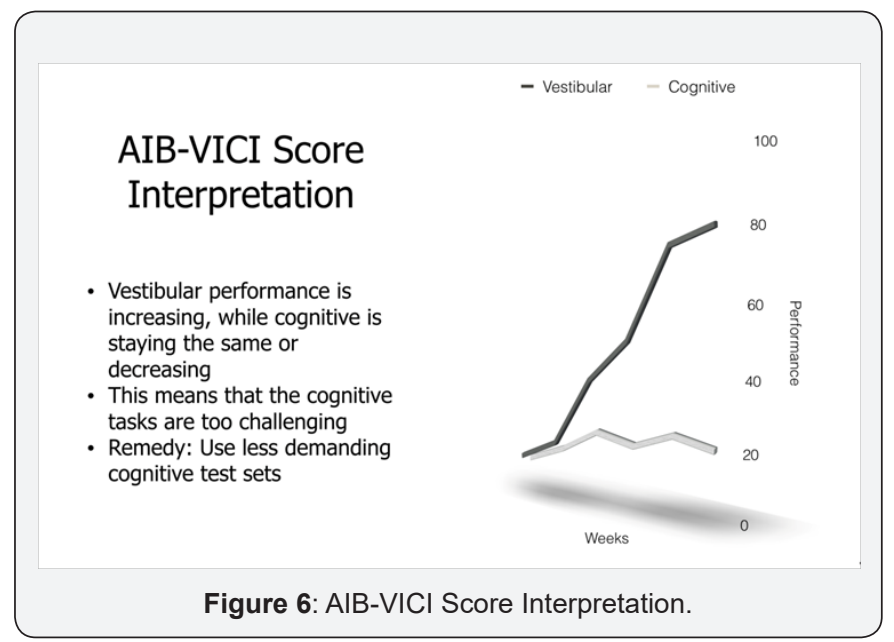

The graph depicts the relationship between vestibular and cognitive performance. In this case, the patient is making progress with vestibular compensation but not with select cognitive performance. Unlike the increased intensity needed for vestibular therapy, cognitive therapy can be negatively influenced by too demanding of tasks. As such, the remedy is to decrease the intensity of the cognitive tasks, or the divided attention task.

B. Second Follow-up

I. Vestibular Assessment

a. Gaze testing: No nystagmus

b. Head Thrust: No corrective saccades with leftward head thrusts

c. Gans SOP testing: fall on condition 6

II. Cognitive Assessment

a. AIB - Vestibular-Cognitive Integration Assessment and Management Tools a) Brief Visuospatial Memory Task (BVMT) - WNL

b) Hopkin's Verbal Learning Task (HVLT) - Improvement

c) NAB Digits Forward/Backward - WNL

d) Stroop Test - WNL

e) Symbol Digit Modalities Test (SDMT) - Improvement

III. Management Strategy

a. VRT for uncompensated left vestibulopathy

i) using hybrid techniques for: substitution, gaze stabilization, adaptation, and habituation protocols focusing on $1 \mathrm{~Hz}-2 \mathrm{~Hz}$ head movements

ii) Cognitive management strategies:

iii) Since BVMT, NAB Digits, and Stroop tests have returned to normal, we can focus on completing exercises for these tasks at the target heart rate of $100 \mathrm{BPM}$

iv) HVLT and SDMT have improved, thus we will increase the HR by 10 BPM and increase the difficulty of the cognitive task as well as the paired vestibular task

\section{Third Follow-up}

I. Vestibular Assessment

a. Gaze testing: No nystagmus

b. Head Thrust: No corrective saccades with leftward head thrusts

c. Gans SOP testing: Normal 1-7

\section{Cognitive Assessment}

AIB - Vestibular-Cognitive Integration Assessment and Management Tools

i. Brief Visuospatial Memory Task (BVMT) - WNL

ii. Hopkin's Verbal Learning Task (HVLT) - WNL

iii. NAB Digits Forward/Backward - WNL

iv. Stroop Test - WNL

v. Symbol Digit Modalities Test (SDMT) - WNL

a. Management Strategy (Self-Directed Maintenance)

i. VRT for uncompensated left vestibulopathy

ii. using hybrid techniques for: substitution, gaze stabilization, adaptation, and habituation protocols focusing on $1 \mathrm{~Hz}-2 \mathrm{~Hz}$ head movements

b. Cognitive management strategies:

i. All cognitive tests have returned to normal; we can focus on completing exercises for these tasks at the target heart rate of 100 BPM (this can be adapted as a self-directed maintenance schedule) (Figure 7) 


\section{Global Journal of Otolaryngology}

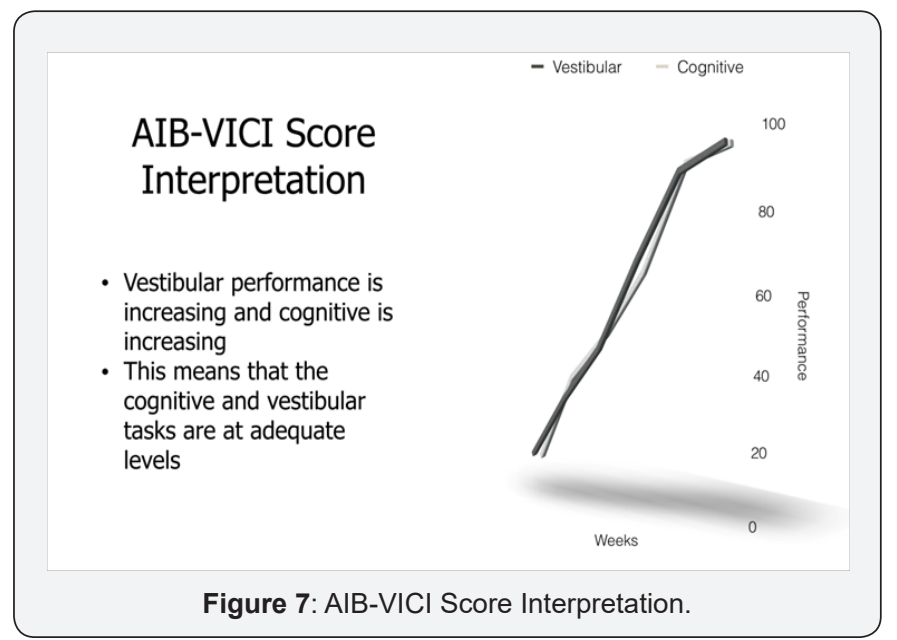

ii. This graph illustrates the intended results of the AIB-VCI. The patient has centrally compensated for the unilateral peripheral vestibulopathy and has reached agecorrected normative cognitive scores. Thus, the patient has been successfully rehabilitated for cortical and labyrinthine aspects of the concussion.

\section{Discussion}

a) In this case, successful cognitive and vestibular management was completed using a strict stepwise progression, which has been shown as a successful concussion rehabilitation strategy [11]. The AIB-VCI recognizes research showing that creating too much cognitive demand, including during therapy sessions, can have devastating and long-lasting effects on the cognitive abilities of the patient, suggesting that adequate rest periods can be of utmost importance in terms of an efficacious rehabilitation strategy [1]. Improper rehabilitation strategies that do not allow for adequate cognitive rest often cause patients to have ongoing symptoms of cognitive decline for many years. This is done in contrast to the intensity needed for effective, and timely, vestibular rehabilitation [12]. Although the cognitive and vestibular tasks are performed together during sessions, they can be manipulated individually, allowing for adjustment of time and intensity of each category.

b) Cortical and labyrinthine concussions have traditionally been managed as a dichotic problem. However, the combination of therapeutic regimes with a single strategy (AIB-VCI) can differentially adjust for difficulty of training sessions on two levels: vestibular task difficulty and cognitive task difficulty. Thus, we can counterbalance for a patient who may need different levels of cognitive versus vestibular training. It has been mentioned previously thattoo intense, or too time-consuming, cognitive load can create a cascade of events in the central nervous system that lead to neuronal cell death [4]. Thus, being able to independently adjust for cognitive and vestibular activities leads to a safe and effective means of cognitive and vestibular rehabilitation post-concussion [13-15].

\section{Conclusion}

Conclusion research is still in its early stages. The AIB-VCI is not intended to be an all-inclusive treatment method. Rather, it is part of an interdisciplinary medical approach towards successful rehabilitation [16-20]. Be it cervical spine injuries, hormone imbalances, visual changes, personality differences, or motor neuron changes, the collaborative approach to concussion management will yield the most benefit to the patient.

\section{References}

1. McLeod Tamara C Valovich, Gerard A Gioia (2010) Cognitive rest: the often neglected aspect of concussion management. Athletic Therapy Today 15(2): 1-3.

2. Cobb S, Battin B (2004) Second-impact syndrome. J Sch Nurs 20(5): 262-267.

3. Barkhoudarian Garni, David A Hovda, Christopher C Giza (2011) The molecular pathophysiology of concussive brain injury. Clin Sports Med 30(1): 33-48.

4. Giza Christopher C, David A Hovda (2001) The neurometabolic cascade of concussion. Journal of athletic training 36(3): 228.

5. Owens Sarah (2016) For Concussion Recovery, Low-Level Activity May Be Better than Rest. Neurology Now.

6. Asken Breton M (2016) Playing Through It: Delayed Reporting and Removal From Athletic Activity After Concussion Predicts Prolonged Recovery. Journal of athletic training 51(4): 329-335.

7. Eyal Bar-Kochba, Mark Scimone, Jonathan Estrada, Christian Franck (2016) Strain and rate-dependent neuronal injury in a 3D in vitro compression model of traumatic brain injury. Scientific Reports 110(6).

8. Malia K (2004) Recommendations for best practice in cognitive rehabilitation therapy: Acquired brain injury. The Society for Cognitive Rehabilitation Therapy 1-57.

9. Leddy John J, John G Baker, Barry Willer (2016) Active Rehabilitation of Concussion and Post-concussion Syndrome. Phys Med Rehabil Clin N Am 27(2): 437-454.

10. Leddy JJ, Kozlowski K, Donnelly JP, Pendergast DR, Epstein LH, et al. (2010) A preliminary study of subsymptom threshold exercise training for refractory post-concussion syndrome. Clin J Sport Med 20(1): 2127.

11. Wallace, Jessica, Tracey Covassin, and Meghan Lafevor (2016) Use of the stepwise progression return-to-play protocol following concussion among practicing athletic trainers. Journal of Sport and Health Science.

12. Lacour Michel, Laurence Bernard-Demanze (2015) Interaction between vestibular compensation mechanisms and vestibular rehabilitation therapy: 10 recommendations for optimal functional recovery. Front Neurol 5: 285

13. Sternberg RJ (2009) Cognitive Psychology. Wadsworth, Belmont, CA, USA, pp. 578.

14. Naomi J Brown, Rebekah C Mannix, Michael J O’Brien, David Gostine, Michael W Collins, et al. (2014) Effect of cognitive activity level on duration of post-concussion symptoms. Pediatrics 133(2): e299-e304.

15. Danny George Thomas, Jennifer N Apps, Raymond G Hoffmann, Michael McCrea, Thomas Hammeke (2015) Benefits of strict rest after acute concussion: a randomized controlled trial. Pediatrics 135(2): 213-223. 
16. Schrank Fredrick A, Nancy Mather, Kevin S McGrew (2014) WoodcockJohnson IV Tests of Cognitive Abilities Examiner's Manual, Standard and Extended Batteries. Itasca, USA

17. Schneider KJ, Iverson GL, Emery CA, McCrory P, Herring SA, et al. (2013) The effects of rest and treatment following sport-related concussion: a systematic review of the literature. Br J Sports Med 47(5): 304-307.

18. McCrory P, Meeuwisse WH, Aubry M, Cantu B, Dvorák J, et al. (2012) Consensus statement on concussion in sport $\mathrm{V}$. The 4th International Conference on Concussion in Sport held in Zurich. Clin J Sport Med 47(5): 250-258.

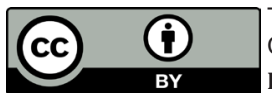

This work is licensed under Creative Commons Attribution 4.0 License

DOI: $10.19080 /$ GJO.2017.05.555653
19. Alsalaheen BA, Whitney SL, Mucha A, Morris LO, Furman JM, et al. (2013) Exercise Prescription Patterns in Patients Treated with Vestibular Rehabilitation After Concussion. Physiother Res Int 18(2): 100-108.

20. McKee AC, Stern RA, Nowinski CJ, Stein TD, Alvarez VE, et al. (2013) The spectrum of disease in chronic traumatic encephalopathy. Brain 136(1): 43-64.

Your next submission with Juniper Publishers will reach you the below assets

- Quality Editorial service

- Swift Peer Review

- Reprints availability

- E-prints Service

- Manuscript Podcast for convenient understanding

- Global attainment for your research

- Manuscript accessibility in different formats

( Pdf, E-pub, Full Text, Audio)

- Unceasing customer service

Track the below URL for one-step submission https://juniperpublishers.com/online-submission.php 